\title{
Sonothrombolysis in the ambulance for ST-elevation myocardial infarction: rationale and protocol
}

\author{
S. El Kadi · T. R. Porter · A. C. van Rossum · O. Kamp
}

Accepted: 20 October 2020 / Published online: 12 November 2020

(C) The Author(s) 2020

\begin{abstract}
Background Treatment of ST-elevation myocardial infarction (STEMI) has improved over the years. Current challenges in the management of STEMI are achievement of early reperfusion and the prevention of microvascular injury. Sonothrombolysis has emerged as a potential treatment for acute myocardial infarction, both for epicardial recanalisation as well as improving microvascular perfusion. This study aims to determine safety and feasibility of sonothrombolysis application in STEMI patients in the ambulance.

Methods Ten patients with STEMI will be included and treated with sonothrombolysis in the ambulance during transfer to the PCI centre. Safety will be assessed by the occurrence of ventricular arrhythmias and shock during sonothrombolysis intervention. Feasibility will be assessed by the extent of protocol completion and myocardial visibility. Efficacy will be determined by angiographic patency rate, ST-elevation resolution, infarct size and left ventricular volumes, and function measured with cardiovascular magnetic resonance imaging, and contrast and strain echocardiography. A comparison will be made with matched controls using an existing STEMI database. Discussion Sonothrombolysis is a novel technique for the treatment of cardiovascular thromboembolic disease. The first clinical trials on its use for STEMI have demonstrated promising results. This study will be the first to examine the feasibility of in-ambulance sonothrombolysis for STEMI.
\end{abstract}

\footnotetext{
S. El Kadi (凶) · A. C. van Rossum · O. Kamp

Department of Cardiology, Amsterdam Cardiovascular

Sciences, Amsterdam UMC Location VUMC, Amsterdam,

The Netherlands

s.elkadi@amsterdamumc.nl

\section{T. R. Porter}

Division of Cardiovascular Medicine, University of Nebraska Medical Center, Omaha, NE, USA
}

Trial registration EU Clinical Trials Register (identifier: 2019-001883-31), registered 2020-02-25.

Keywords Sonothrombolysis · ST-elevation myocardial infarction · Ambulance $\cdot$ Treatment

\section{Background}

ST-segment elevation on the electrocardiogram (ECG) indicates the occlusion of an epicardial coronary artery, most commonly due to thrombus formation at the site of a ruptured atherosclerotic plaque. The optimal treatment strategy for ST-elevation myocardial infarction (STEMI) is immediate restoration of epicardial coronary blood flow. Currently, primary percutaneous coronary intervention (PCI) with concomitant dual antiplatelet therapy and periprocedural anticoagulation constitutes the treatment of choice in STEMI patients [1]. While successful reperfusion has led to a marked decrease in annual deaths over the past decades, long-term mortality and morbidity remain substantial [2, 3]. In addition to prolonged ischaemia and subsequent infarct size, microvascular injury due to ischaemic and reperfusion processes is a major determinant of adverse left ventricular remodelling and poor prognosis [4] and occurs in up to $50 \%$ of the patients [5]. By minimising infarct size and microvascular injury, left ventricular function may be preserved with improved long-term prognosis. Several studies have focused on early therapeutic interventions for reduction of infarct size and microvascular injury. Early drug therapy strategies include the use of low-dose fibrinolysis [6, 7], beta-blockers [8], adenosine [9], cyclosporine [10] and complement inhibition [11]. No or only small beneficial effects in a selected patient population have been demonstrated. Other interventions such as thrombus aspiration to reduce distal embolisation [12] and remote ischaemic 
Table 1 Summary of recent randomised controlled trials on pre-hospital therapeutic strategies before PCl in patients with acute myocardial infarction

\begin{tabular}{|c|c|c|c|c|}
\hline Therapy & Study & $\begin{array}{l}\text { No. of } \\
\text { patients }\end{array}$ & Primary endpoints & Outcome \\
\hline \multirow[t]{3}{*}{ Abciximab } & Pels et al. (2008) [34] & 101 & $\begin{array}{l}\text { STR before PCI } \\
\text { Initial TIMI } 3 \text { flow in IRA }\end{array}$ & $\begin{array}{l}\text { No improvement in initial TIMl flow or } \\
\text { STR }\end{array}$ \\
\hline & Petronio et al. (2012) [35] & 110 & Infarct size (LGE-MRI) & No reduction in infarct size \\
\hline & Ohlmann et al. (2012) [36] & 256 & Complete STR (>70\%) after PCl & No improvement in STR \\
\hline \multirow[t]{2}{*}{ Tirofiban } & Van 't Hof (2008) [37] & 984 & Mean residual STD after $\mathrm{PCl}$ & Improvement in STR \\
\hline & El Khoury et al. (2010) [38] & 320 & Initial TIMI 2/3 flow in IRA & No improvement in initial TIMI 2/3 flow \\
\hline Clopidogrel & Zeymer et al. (2012) [39] & 337 & Initial TIMI 2/3 flow in IRA & No improvement in initial TIMI 2/3 flow \\
\hline Ticagrelor & Montalescot et al. (2014) [40] & 1862 & $\begin{array}{l}\text { Initial TIMI flow } \\
\text { STR before PCI }\end{array}$ & $\begin{array}{l}\text { No improvement in initial TIMI flow or } \\
\text { STR }\end{array}$ \\
\hline Bivalirudin & Steg et al. (2013) [41] ${ }^{\mathrm{a}}$ & 2218 & $\begin{array}{l}\text { Combined endpoint of death or major bleeding } \\
\text { ( } 30 \text { days) }\end{array}$ & $\begin{array}{l}\text { Reduced composite endpoint (driven by } \\
\text { less major bleeding), increased stent } \\
\text { thrombosis }\end{array}$ \\
\hline \multirow[t]{2}{*}{ Tenecteplase } & Armstrong (2006) [42] & 304 & $\begin{array}{l}\text { Combined endpoint of death, re-infarction, refrac- } \\
\text { tory ischaemia, congestive heart failure, cardio- } \\
\text { genic shock, and major ventricular arrhythmia }\end{array}$ & $\begin{array}{l}\text { No difference in combined endpoint } \\
\text { compared with PCl alone }\end{array}$ \\
\hline & Thiele et al. (2011) [43] & 162 & Infarct size (LGE-MRI) & No reduction in infarct size \\
\hline \multirow[t]{2}{*}{ Metoprolol } & Ibanez et al. (2013) [44] ${ }^{\mathrm{a}}$ & 270 & Infarct size (LGE-MRI) & Reduction in infarct size \\
\hline & Roolvink et al. (2016) [8] & 684 & Infarct size (LGE-MRI) & No reduction in infarct size \\
\hline \multirow[t]{2}{*}{ Oxygen } & Stub et al. (2015) [45] & 441 & Infarct size (enzymes) & Increase in infarct size \\
\hline & Khoshnood et al. (2018) [46] & 95 & Myocardial salvage index (LGE-MRI) & No improvement of myocardial salvage \\
\hline $\begin{array}{l}\text { Ischaemic } \\
\text { conditioning }\end{array}$ & Hausenloy et al. (2019) [47] ${ }^{\mathrm{a}}$ & 5115 & $\begin{array}{l}\text { Combined endpoint of cardiac death and HF hospi- } \\
\text { talisation }\end{array}$ & No improvement of clinical outcomes \\
\hline Hypothermia & Testori et al. (2019) [48] & 101 & Myocardial salvage index (LGE-MRI) & No improvement of myocardial salvage \\
\hline
\end{tabular}

conditioning [13] to reduce the possible detrimental effects of spontaneous reperfusion likewise did not improve outcome. A growing number of studies on adjunctive therapies for STEMI are performed in the pre-hospital setting. The time from STEMI diagnosis to PCI provides a window of opportunity for early therapy. A summary of recent studies on prehospital therapeutic strategies before PCI to reduce infarct size in STEMI patients and improve clinical outcomes is listed in Tab. 1. Recently, sonothrombolysis has been proposed as an adjunctive therapy for acute myocardial infarction [14]. In-vitro studies and in-vivo STEMI models have shown the potential of sonothrombolysis for restoration of epicardial and microvascular flow [15]. A recent randomised controlled trial on the effects of pre- and post-PCI sonothrombolysis in STEMI patients demonstrated a higher angiographic recanalisation rate prior to PCI and reduced infarct size in the intervention group [16]. Pre-PCI sonothrombolysis was performed at the emergency department in the hospital and resulted in thrombolysis in myocardial infarction (TIMI) flow grade $\geq 2$ in almost half of the patients. By performing sonothrombolysis even before hospital arrival, its efficacy may be further improved. In this study, safety and technical feasibility of sonothrombolysis in STEMI patients in the pre-hospital ambulance setting will be assessed. To our knowledge, this is the first time that therapeutic echocardiography will be used in the ambulance.

\section{Methods}

\section{Objectives}

The primary objective is to study safety and feasibility of sonothrombolysis application in the ambulance. The secondary objective is to evaluate the efficacy of in-ambulance sonothrombolysis as assessed by STelevation resolution, initial angiographic patency, infarct characteristics and left ventricular dimensions, function, perfusion and remodelling.

\section{Endpoints}

The main endpoints are safety and practical feasibility. Safety will be assessed by the occurrence of ventricular arrhythmias defined as sustained ventricular tachycardia and/or ventricular fibrillation and the occurrence of shock defined as a systolic blood pressure $<100 \mathrm{mmHg}$ in combination with tachycardia (heart rate $>100 / \mathrm{min}$ ), after initiation of sonothrombolysis and before PCI.

These endpoints will provide relevant information regarding the safety of sonothrombolysis in the ambulance. Two forms of shock are relevant for the present 


\begin{tabular}{|c|c|}
\hline $\begin{array}{l}\text { Inclusion } \\
\text { criteria }\end{array}$ & $\begin{array}{l}\text { - Acute (within } 12 \mathrm{~h} \text { ) or worsening chest pain or shortness of } \\
\text { breath associated with }>1.0 \mathrm{mV} \text { ST elevation on the ECG in } \\
\text { at least } 2 \text { contiguous leads } \\
\text { - Age } \geq 30 \text { years } \\
\text { - Adequate apical and/or parasternal images by echocardiog- } \\
\text { raphy }\end{array}$ \\
\hline $\begin{array}{l}\text { Exclusion } \\
\text { criteria }\end{array}$ & $\begin{array}{l}\text { - Previous coronary bypass surgery } \\
\text { - Cardiogenic shock } \\
\text { - Known or suspected hypersensitivity to ultrasound contrast } \\
\text { agent used for the study } \\
\text { - Known bleeding diathesis or contraindication to glycopro- } \\
\text { tein IIB/IIIA inhibitors, anticoagulants or aspirin } \\
\text { - Known large right to left intracardiac shunts }\end{array}$ \\
\hline
\end{tabular}

study: anaphylactic shock (very rare in case of commercially available microbubbles, 1:10,000-100,000) and cardiogenic shock. The incidence of cardiogenic shock in STEMI patients is around 7\% [17]. The incidence of ventricular arrhythmias in STEMI patients before percutaneous coronary intervention is around $3 \%$ [18]. It is important to note that some ventricular arrhythmias, e.g. runs of ventricular premature complexes or accelerated idiopathic ventricular arrhythmias, are not uncommon after flow restoration in STEMI and are even related to improvement of myocardial perfusion [19].

Practical feasibility will be assessed by the extent of sonothrombolysis completion during ambulance transfer. The ultrasound images will be saved for offline review; sonothrombolysis completion will be measured by counting the number of high mechanical index impulses applied in each view and the quality of the acquired apical views will be scored.

Secondary endpoints are ST-elevation resolution, initial TIMI flow, infarct characteristics and left ventricular dimensions and function at baseline (3-5 days) and follow-up (6-8 weeks) with cardiovascular magnetic resonance imaging (CMR). Myocardial perfusion and left ventricular remodelling parameters will be assessed on follow-up contrast-enhanced echocardiography (3-4 months). Six-month eventfree survival will be calculated from treatment initiation to 6 months afterwards, where events include death, congestive heart failure, life-threatening arrhythmias, recurrence of acute coronary syndrome and need for prophylactic defibrillator.

\section{Patient enrolment}

This study will be conducted in accordance with the standards for Good Clinical Practice and the Declaration of Helsinki. Ethics approval was obtained on 3 February 2020 from the Medical Ethics Review Committee VUmc (reference: 2019.556-NL69980.029.19). Given the logistical challenge of the study and the primary aim to assess feasibility, a total of ten patients will be included. Due to the urgent setting and need for rapid initiation of the study protocol, only brief oral consent will be obtained. The written informed consent procedure will be deferred until after coronary angiography. Inclusion and exclusion criteria are listed in Tab. 2. All patients will receive drug therapy consisting of dual antiplatelet therapy (DAPT) and intravenous heparin in the ambulance. DAPT will be continued for 1 year according to current guidelines.

\section{Study procedures}

The study will be conducted by the Amsterdam University Medical Center-location VUmc in collaboration with the emergency medical services (EMS) in Zaandam, the Netherlands. In the ambulance, ECGs of patients with signs of STEMI will be faxed to the PCI centre via the Lifenet system [20]. When STEMI diagnosis is confirmed and patient is accepted for coronary angiography with optional PCI, eligible patients will be asked to give oral informed consent and will be included in the trial (Fig. 1, Study flow chart). Sonothrombolysis will be performed by a trained medical doctor (SK) in the ambulance and applied for $20 \mathrm{~min}$ or until hospital arrival. The treatment consists of commercially available microbubble infusion ( $1.5 \mathrm{ml}$ Luminity, diluted in $50 \mathrm{ml} \mathrm{NaCl}$; Lantheus MI, Newbury, United Kingdom) and simultaneous transthoracic echocardiography with a Philips CX-50 ultrasound machine built into the ambulance (Fig. 2). Low mechanical index imaging $(\mathrm{MI}<0.3)$ will be used to visualise the myocardium within each view and to monitor for replenishment within each of the segments after application of high MI. Multiple (>10) intermittent high MI impulses (20 frames 1.0-1.2 MI; transmit frequency $1.6 \mathrm{MHz}$; pulse duration $<5 \mu \mathrm{s}$ ) will be administered in either three apical windows or parasternal short-axis windows, depending on image quality. A total of 30-60 impulses will be given, with 4-8 sec between consecutive high MI impulses to allow for replenishment of contrast within the perfusion beds. The total duration of sonothrombolysis will be measured as well as total number of applied flashes in each view. Off-site quality analysis of the images will be performed in which the in-plane visibility of the myocardium will be recorded and scored by an experienced blinded core lab.

At least three ECGs will be performed: before and after sonothrombolysis and after primary PCI. Absolute and relative cumulative ST-elevation resolution will be measured. Creatine kinase (CK), creatine kinase MB (CK-MB) and troponin levels will be obtained through standard care venous blood sampling in the hospital. Maximum biomarker levels will be reported.

All patients will undergo invasive coronary angiography. Primary PCI will be performed according to the guidelines and at the discretion of the interventional cardiologist. TIMI flow grade before and after PCI, myocardial blush grade and thrombus burden will be reported.

CMR imaging will be performed at baseline (3-5 days after presentation) and 6 to 8 weeks later. 
Fig. 1 Study flow chart. (STEMI ST-elevation myocardial infarction, EMS emergency medical services, CAG coronary angiography, $\mathrm{PCl}$ percutaneous coronary intervention)

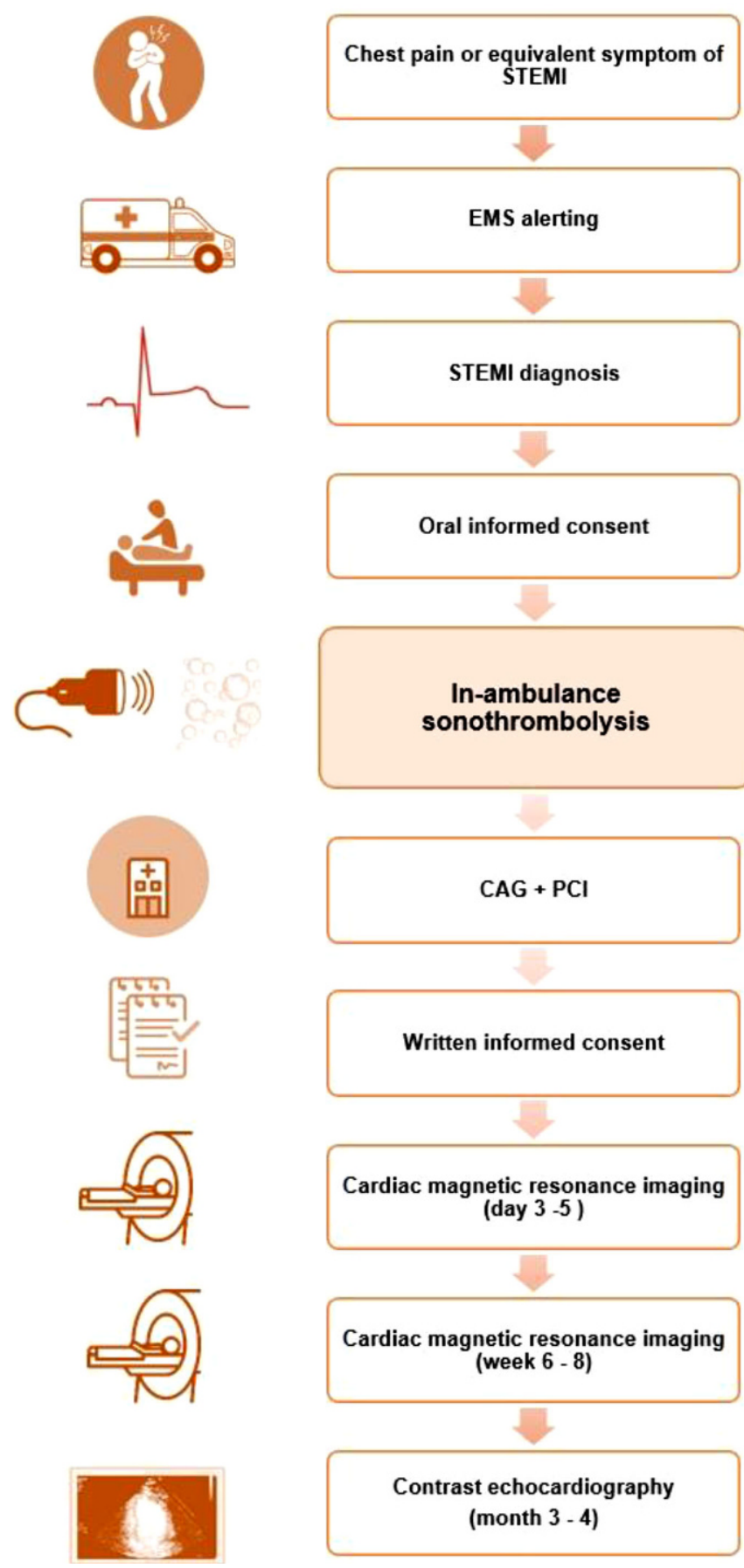

Pre-hospital

In-patient care
A clinical 1.5 or $3.0 \mathrm{~T}$ scanner with phased-array cardiac receiver coils will be used. Cine images are obtained using a steady state free precession sequence in three long-axis views and multiple short-axis views to examine left ventricular mass, function and volumes and to quantify left ventricular ejection fraction. T2-weighted images will be taken in short-axis planes identical to the cine images. T1 mapping in three parallel short-axis views positioned at the basal, mid and apical levels will be done using a Shortened Modified Look-Locker Inversion recovery sequence. First-pass perfusion will be performed in the same three short- axis views as $\mathrm{T} 1$ mapping using saturation recovery imaging. Early and late gadolinium enhanced (LGE) images are acquired 2 and $10 \mathrm{~min}$, respectively, postinjection using a segmented gradient echo sequence. LGE images will be used to assess infarct size, the presence and extent of microvascular injury and to measure the area at risk. Short-axis T2-weighted images will be used for the detection of intramyocardial haemorrhage and infarct-related oedema. All CMR analyses will be performed in a core laboratory by blinded observers. 


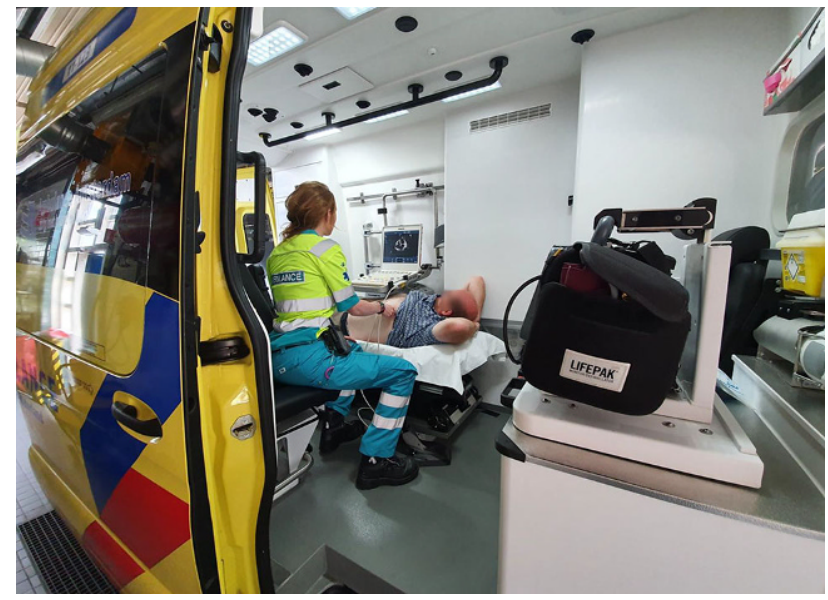

Fig. 2 Portable ultrasound system mounted inside the ambulance enables diagnostic and therapeutic echocardiography (sonothrombolysis) during patient transfer to the $\mathrm{PCl}$ centre

Contrast-enhanced echocardiography will be performed 3-4 months after initial presentation. Left ventricular function and volumes will be measured. Left ventricular remodelling will be defined as a $20 \%$ increase in end-diastolic volume at the 3 month followup biplane contrast-enhanced echocardiogram compared with the pre-discharge echocardiogram.

Follow-up will take place at 6 months by telephone contact and electronic patient records will be consulted.

\section{Statistical analysis}

Statistical analyses for the primary endpoints will be purely descriptive. Baseline characteristics will be presented, continuous variables will be given as $n$ (\%) and mean with interquartile range (IQR). Secondary outcomes will be compared between patients included in this study and historical control patients. All these analyses will be considered exploratory. Categorical variables will be compared between groups using a chi-square test or Fisher's exact test. Continuous variables will be compared using an independent sample t-test (if normally distributed) or the MannWhitney test (otherwise). The study and the trial conduct will be audited by an independent monitor from the Amsterdam UMC.

\section{Challenges}

Although multiple studies on new therapeutic strategies for STEMI have been performed in the pre-hospital setting (Tab. 1), incorporation of sonothrombolysis in the ambulance is more challenging.

It requires preparation of all materials and processes, briefing of EMS nurses and staff of the emergency control room as well as the presence of a trained physician to perform sonothrombolysis. The aim is to complete the entire process-from oral informed consent and microbubble preparation to ultrasound imaging and sonothrombolysis-during patient transfer. Obtaining oral consent, preparing the microbubble solution and obtaining appropriate ultrasound views is estimated to take $4 \mathrm{~min}$. The ultrasound regimen consists of 30-60 high MI impulses and can be completed within $8 \mathrm{~min}$, considering a $4-8 \mathrm{sec} \mathrm{mi}-$ crobubble replenishment time in between the high MI impulses. To prevent any delay in patient transfer, the study treatment needs to be completed within 10-15 min, which approximates the mean duration of the ambulance ride to the PCI centre. A custom-built holder has been developed to keep the ultrasound machine in place (Fig. 2). Furthermore, since Luminity microbubbles will be used, a mobile cooling device is necessary to comply to the recommended storage conditions. Since only one vehicle is equipped with the appropriate sonothrombolysis equipment, recruitment time might be long. To overcome this difficulty, the ambulance control room will be notified about the study and requested to assign patients with possible STEMI (e.g. patients with chest pain) to the equipped vehicle.

\section{Discussion}

Thrombolysis and PCI have significantly improved the prognosis of STEMI patients. However, two major clinical problems remain. Firstly, early reperfusion is restricted by patient factors, delays in transfer to PCI capable centres and delays in PCI initiation [1]. The longer the ischaemic time, the greater the size and transmurality of the infarct zone [21, 22]. Actions have been taken to reduce ischaemic time by facilitating early STEMI diagnosis and prehospital activation of the cardiac catheterisation lab. However, total ischaemic time in STEMI patients remains substantial [23]. Secondly, although successful epicardial revascularisation may be achieved, up to $50 \%$ of the patients exhibit microvascular injury, resulting in higher infarct sizes, adverse left ventricle remodelling and worse prognosis [4]. Novel therapeutic interventions for STEMI should preferably be applied within minutes of STEMI diagnosis and should also treat or prevent microvascular injury.

In recent years, sonothrombolysis has emerged as a potential adjunctive therapy in cardiovascular ischaemic disease. The technique is based on the lytic and flow-inducing properties of the ultrasoundinduced cavitation of contrast microspheres. Once high MI ultrasound impulses are administered during simultaneous infusion of ultrasound contrast agents, the contrast microspheres compress and expand and eventually collapse, inducing local microstreaming capable of thrombus dissolution (Fig. 3; [24, 25]). Various animal models have been used to examine therapeutic contrast echocardiography with and without fibrinolytic drugs [26-28]. Xie et al. found 
Fig. 3 Restoration of epicardial and microvascular blood flow in ST-elevation myocardial infarction using sonothrombolysis. (NO nitric oxide, ATP adenosine triphosphate)

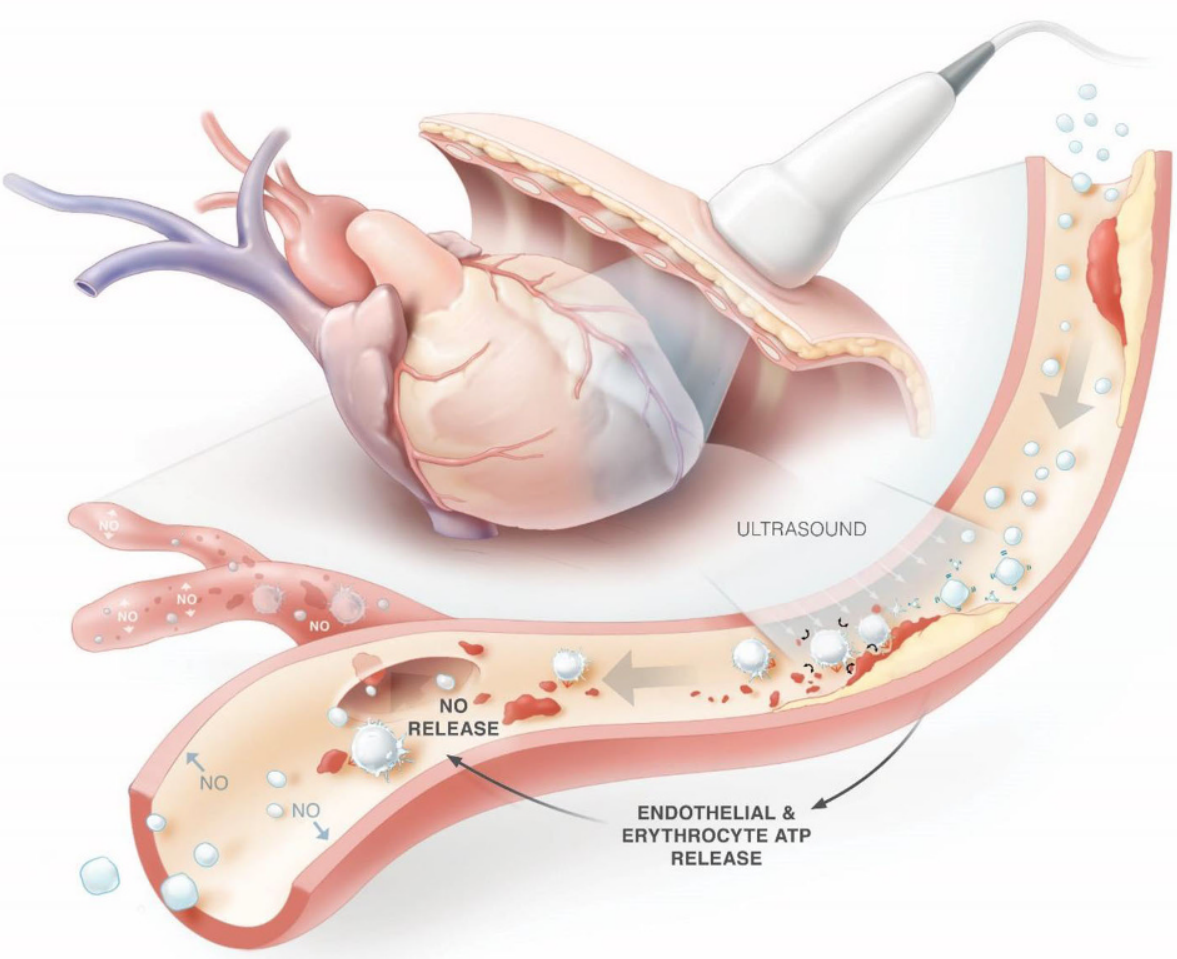

unexpectedly that high MI impulses using a diagnostic transducer in combination with microbubbles in pigs with acute left anterior descending occlusions not only resulted in higher epicardial recanalisation rates but also resulted in ST-segment resolution and corresponding recovery of wall thickening in six pigs $(40 \%)$ that did not have epicardial recanalisation [29]. Improvement of microvascular flow could be related to sonolysis of microvascular thrombi or the increased release of the vasodilator and antiinflammatory nitric oxide from the microvascular endothelium (Fig. 3). The latter explanation is supported by a study in mice where augmentation of limb perfusion was observed upon ultrasound and microbubbles application, whereas inhibition of endothelial nitric oxide synthase attenuated the flow augmentation by $70 \%$ [30]. The first pilot study on clinical application of ultrasound and microbubbles in STEMI patients showed safety and feasibility of pre-PCI sonothrombolysis [31]. As preclinical studies suggested efficacy of thrombus dissolution could be improved by increasing high MI pulse duration [32], a small trial was performed using $20 \mu$ s high MI impulses [33]. While feasible for restoring microvascular perfusion, transient focal vasoconstriction was seen in three of six patients. In the subsequent MRUSMI trial, 100 patients were randomised to either diagnostic ultrasound-guided treatment with intermittent high MI impulses $(5 \mu \mathrm{s})$ during microbubble infusion or to a control group [16]. Treatment was given preand post-PCI and lasted approximately $15 \mathrm{~min}$ per treatment. Angiographic recanalisation prior to PCI was achieved in $48 \%$ in the treatment group versus $20 \%$ in the control group. Positive results were observed regarding infarct size, left ventricular ejection fraction and need for defibrillator implantation, while vasoconstriction was not observed. Currently, several trials are ongoing that aim to investigate the effects of post-PCI sonothrombolysis on microvascular injury and infarct size (MRUSMI, EU Clinical Trials Register 2018-001277-24).

Since contrast ultrasound is portable, readily available and non-invasive, this technique can be provided at the point-of-care to the STEMI patient. To prevent system delay, sonothrombolysis in the ambulance may offer a convenient solution to achieve early myocardial reperfusion and concomitantly treat microvascular injury in the setting of a STEMI. This study will be the first study in the ambulance setting to examine feasibility of pre-hospital therapeutic contrast ultrasound in STEMI patients.

\section{Trial status}

Recruitment is expected to start in September 2020, completion of recruitment is expected in December 2020. The date and version of the study protocol approved by the ethical review board are: version 3, January 2020.

Acknowledgements The authors would like to thankA. Siegers (medical manager of the Ambulance Post Zaandam) and M. Zanstra (coordinating ambulance nurse of the Ambulance Post Zaandam) for their willingness to participate in the study and their contributions to the logistics of the study, 
and Stephanie Philippaerts for designing the illustration in Fig. 3.

Funding This research received no specific grant from any funding agency in the public, commercial or not-for-profit sectors.

Conflict of interest T.R. Porter Porter receives research equipment support from Philips Ultrasound. From Bracco, Dr. Porter receives educational support and he is on their Speaker's Bureau. Dr. Porter also receives salary and research support from the Theodore F. Hubbard Foundation. In addition, Dr. Porter has a patent issued on Thrombolytic Agents and Methods of Treatment for Thrombosis (issued to \#6,197,345). S. El Kadi, A.C. van Rossum and O. Kamp declare that they have no competing interests.

Open Access This article is licensed under a Creative Commons Attribution 4.0 International License, which permits use, sharing, adaptation, distribution and reproduction in any medium or format, as long as you give appropriate credit to the original author(s) and the source, provide a link to the Creative Commons licence, and indicate if changes were made. The images or other third party material in this article are included in the article's Creative Commons licence, unless indicated otherwise in a credit line to the material. If material is not included in the article's Creative Commons licence and your intended use is not permitted by statutory regulation or exceeds the permitted use, you will need to obtain permission directly from the copyright holder. To view a copy of this licence, visit http://creativecommons.org/licenses/by/4.0/.

\section{References}

1. Ibanez B, James S, Agewall S, et al. ESC Guidelines for the management of acute myocardial infarction in patients presenting with ST-segment elevation: The Task Force for the management of acute myocardial infarction in patients presenting with ST-segment elevation of the European Society of Cardiology (ESC). Eur HeartJ. 2017;2018:119-77.

2. Pedersen F, Butrymovich V, KelbaekH, etal. Short- andlongterm cause of death in patients treated with primary PCI for STEMI. JAm Coll Cardiol. 2014;64:2101-8.

3. Torabi A, Cleland JG, Khan NK, et al. The timing of developmentandsubsequentclinical course ofheartfailure after a myocardial infarction. Eur Heart J. 2008;29:859-70.

4. van Kranenburg M, Magro M, Thiele H, et al. Prognostic value of microvascular obstruction and infarct size, as measured by CMR in STEMI patients. JACC Cardiovasc Imaging. 2014;7:930-9.

5. Carrick D, Haig C, Rauhalammi S, et al. Prognostic significance of infarct core pathology revealed by quantitative non-contrastincomparison with contrastcardiacmagnetic resonance imaging in reperfused ST-elevation myocardial infarction survivors. Eur Heart J. 2016;37:1044-59.

6. Assessment of the S, Efficacy of a New Treatment Strategy with Percutaneous Coronary Intervention i. Primary versus tenecteplase-facilitated percutaneous coronary intervention in patients with ST-segment elevation acute myocardial infarction (ASSENT-4 PCI): randomised trial. Lancet. 2006;367:569-78.

7. McCartney PJ, Eteiba H, Maznyczka AM, et al. Effect of Low-Dose Intracoronary Alteplase During Primary Percutaneous Coronary Intervention on Microvascular Obstruction in Patients With Acute Myocardial Infarction: A Randomized Clinical Trial. JAMA. 2019;321:56-68.
8. RoolvinkV, Ibanez B, Ottervanger JP, et al. Early Intravenous Beta-Blockers in Patients With ST-Segment Elevation Myocardial Infarction Before Primary Percutaneous Coronary Intervention. JAm Coll Cardiol. 2016;67:2705-15.

9. Nazir SA, McCann GP, Greenwood JP, et al. Strategies to attenuate micro-vascular obstruction during P-PCI: the randomized reperfusionfacilitated bylocal adjunctive therapy in ST-elevation myocardial infarction trial. Eur Heart J. 2016;37:1910-9.

10. CungT-T, MorelO, Cayla G, et al. Cyclosporine before PCI in Patients with Acute Myocardial Infarction. New Engl J Med. 2015;373:1021-31.

11. Investigators AA, Armstrong PW, Granger CB, et al. Pexelizumab for acute ST-elevation myocardial infarction in patients undergoing primary percutaneous coronary intervention: a randomized controlled trial. JAMA. 2007;297:43-51.

12. Elgendy IY, Huo T, Bhatt DL, Bavry AA. Is Aspiration Thrombectomy Beneficial in Patients Undergoing Primary Percutaneous Coronary Intervention? Meta-Analysis of Randomized Trials. Circ Cardiovasc Interv. 2015;8:e2258.

13. Hausenloy DJ, Kharbanda RK, Møller UK, et al. Effect of remote ischaemic conditioning on clinical outcomes in patients with acute myocardial infarction (CONDI-2/ERICPPCI): a single-blind randomised controlled trial. Lancet. 2019;394:1415-24.

14. Slikkerveer J, Juffermans LJM, van Royen N, Appelman Y, Porter TR, Kamp O. Therapeutic application of contrast ultrasound in ST elevation myocardial infarction: Role in coronary thrombosis and microvascular obstruction. Eur Heart JAcute Cardiovasc Care. 2019;8:45-53.

15. Xie F, Gao S, Wu J, et al. Diagnostic ultrasound induced inertial cavitation to non-invasively restore coronary and microvascular flow in acute myocardial infarction. Plos One. 2013;8:e69780.

16. Mathias W Jr., Tsutsui JM, Tavares BG, et al. Sonothrombolysis in ST-Segment Elevation Myocardial Infarction Treated With Primary Percutaneous Coronary Intervention. J Am Coll Cardiol. 2019;73:2832-42.

17. Anderson ML, Peterson ED, Peng SA, et al. Differences in the profile, treatment, and prognosis of patients with cardiogenic shock by myocardial infarction classification a report from NCDR. Circ Cardiovasc Qual Outcomes. 2013;6:708-15.

18. Kosmidou I, Embacher M, McAndrew T, et al. Early Ventricular Tachycardia or Fibrillation in Patients With ST Elevation Myocardial Infarction Undergoing Primary Percutaneous Coronary Intervention and Impact on Mortality and Stent Thrombosis (from the Harmonizing Outcomes with Revascularization and Stents in Acute Myocardial Infarction Trial). Am J Cardiol. 2017;120:1755-60.

19. Majidi M, Kosinski AS, Al-Khatib SM, et al. Reperfusion ventricular arrhythmia 'bursts' in TIMI 3 flow restoration with primary angioplasty for anterior ST-elevation myocardial infarction: a more precise definition of reperfusion arrhythmias. Europace. 2008;10:988-97.

20. Sejersten M, Sillesen M, Hansen PR, et al. Effect on treatment delay of prehospital teletransmission of 12-lead electrocardiogram to a cardiologist for immediate triage and direct referral of patients with ST-segment elevation acute myocardial infarction to primary percutaneous coronary intervention. Am J Cardiol. 2008;101:941-6.

21. Reimer KA, Lowe JE, Rasmussen MM, Jennings RB. The wavefront phenomenon of ischemic cell death. 1. Myocardial infarct size vs duration of coronary occlusion in dogs. Circulation. 1977;56:786-94. 
22. Reimer KA, The JRB. "wavefront phenomenon" of myocardial ischemic cell death. II. Transmural progression of necrosis within the framework of ischemic bed size (myocardium at risk) and collateral flow. Lab Invest. 1979;40:633-44.

23. Vos NS, Amoroso G, Grundeken MJ, et al. Pre-hospital management, procedural performance and outcomes for primary percutaneous coronary intervention in ST-elevation myocardial infarction in the Netherlands: Insights from the Dutch cohort of the APPOSITION-III trial. Neth Heart J. 2016;24:730-9.

24. Chen X, Leeman JE, Wang J, Pacella JJ, Villanueva FS. New insights into mechanisms of sonothrombolysis using ultrahigh-speed imaging. Ultrasound Med Biol. 2014;40:258-62.

25. Marmottant P, Hilgenfeldt S. Controlled vesicle deformation and lysis by single oscillating bubbles. Nature. 2003;423:153-6.

26. Hitchcock KE, Ivancevich NM, Haworth KJ, et al. Ultrasound-enhanced rt-PA thrombolysis in an ex vivo porcine carotid artery model. Ultrasound Med Biol. 2011;37:1240-51.

27. Brown AT, Flores R, Hamilton E, Roberson PK, Borrelli MJ, CulpWC. Microbubbles improve sonothrombolysis in vitro and decrease hemorrhage in vivo in a rabbit stroke model. Invest Radiol. 2011;46:202-7.

28. Xie F, Lof J, Everbach C, et al. Treatment of acute intravascular thrombi with diagnostic ultrasound and intravenous microbubbles. JACC Cardiovasc Imaging. 2009;2:511-8.

29. Xie F, Lof J, Matsunaga T, Zutshi R, Porter TR. Diagnostic ultrasound combined with glycoprotein IIb/IIIa-targeted microbubbles improves microvascular recovery after acute coronary thrombotic occlusions. Circulation. 2009;119:1378-85.

30. Belcik JT, Mott BH, Xie A, et al. Augmentation of limb perfusion and reversal of tissue ischemia produced by ultrasound-mediated microbubble cavitation. Circ Cardiovasc Imaging. 2015;8:e2979.

31. Slikkerveer J, Kleijn SA, Appelman Y, et al. Ultrasound enhanced prehospital thrombolysis using microbubbles infusion in patients with acute ST elevation myocardial infarction: pilot of the Sonolysis study. Ultrasound Med Biol. 2012;38:247-52.

32. Wu J, Xie F, Kumar T, et al. Improved sonothrombolysis from a modified diagnostic transducer delivering impulses containing a longer pulse duration. Ultrasound Med Biol. 2014;40:1545-53.

33. Roos ST, Juffermans LJ, van Royen N, etal. Unexpected High Incidence of Coronary Vasoconstriction in the Reduction of Microvascular Injury Using Sonolysis (ROMIUS) Trial. Ultrasound Med Biol. 2016;42:1919-28.

34. Pels K, Schroder J, Witzenbichler B, et al. Prehospital versus periprocedural abciximab in ST-elevation myocardial infarction treated by percutaneous coronary intervention. EurJEmerg Med. 2008;15:324-9.

35. Petronio AS, De Carlo M, Strata E, etal. Impact of early abciximab administration on infarct size in patients with ST-elevation myocardial infarction. Int J Cardiol. 2012;155:230-5.

36. Ohlmann P, Reydel P, Jacquemin L, et al. Prehospital abciximab in ST-segment elevation myocardial infarction: results of the randomized, double-blind MISTRAL study. Circ Cardiovasc Interv. 2012;5:69-76:S1.

37. Van't Hof AW, Ten Berg J, Heestermans T, et al. Prehospital initiation of tirofiban in patients with ST-elevation myocardial infarction undergoing primary angioplasty (On-TIME 2): a multicentre, double-blind, randomised controlled trial. Lancet. 2008;372:537-46.

38. El Khoury C, Dubien PY, Mercier C, et al. Prehospital highdose tirofiban in patients undergoing primary percutaneous intervention. The AGIR-2 study. Arch Cardiovasc Dis. 2010;103:285-92.

39. Zeymer U, Arntz HR, Mark B, et al. Efficacy and safety of a high loading dose of clopidogrel administered prehospitally to improve primary percutaneous coronary intervention in acute myocardial infarction: the randomized CIPAMI trial. Clin Res Cardiol. 2012;101:305-12.

40. Montalescot G, van 't Hof AW. Prehospital ticagrelor in STsegment elevation myocardial infarction. N Engl J Med. 2014;371:2339.

41. Steg PG, van 't Hof A, Hamm CW, et al. Bivalirudin started during emergency transport for primary PCI. N Engl J Med. 2013;369:2207-17.

42. Armstrong PW. the WEST Steering Committee. A comparison of pharmacologic therapy with/without timely coronary intervention vs. primary percutaneous intervention early after ST-elevation myocardial infarction: the WEST (Which Early ST-elevation myocardial infarction Therapy) study. Eur HeartJ. 2006;27:1530-8.

43. ThieleH, Eitel I, MeinbergC, etal. Randomized comparison of pre-hospital-initiated facilitated percutaneous coronary intervention versus primary percutaneous coronary intervention in acute myocardial infarction very early after symptom onset: theLIPSIA-STEMI trial (Leipzigimmediate prehospital facilitated angioplasty in ST-segment myocardial infarction). JACC Cardiovasc Interv. 2011;4:605-14.

44. Ibanez B, Macaya C, Sanchez-Brunete V, et al. Effect of early metoprolol on infarct size in ST-segmentelevation myocardial infarction patients undergoing primary percutaneous coronary intervention. Circulation. 2013;128:1495-503. the Effect of Metoprolol in Cardioprotection During an Acute Myocardial Infarction (METOCARD-CNIC) trial.

45. Stub D, Smith K, Bernard S, et al. Air Versus Oxygen in ST-Segment-Elevation Myocardial Infarction. Circulation. 2015;131:2143-50.

46. Khoshnood A, Akbarzadeh M, Carlsson M, et al. Effect of oxygen therapy on chest pain in patients with ST elevation myocardial infarction: results from the randomized SOCCER trial. Scand Cardiovasc J. 2018;52:69-73.

47. Hausenloy DJ, Kharbanda RK, Moller UK, et al. Effect of remote ischaemic conditioning on clinical outcomes in patients with acute myocardial infarction (CONDI-2/ERICPPCI): a single-blind randomised controlled trial. Lancet. 2019;394:1415-24.

48. Testori C, Beitzke D, Mangold A, et al. Out-of-hospital initiation of hypothermia in ST-segment elevation myocardial infarction: a randomised trial. Heart. 2019;105:531-7. 